\title{
Artículos
}

\section{Asentamientos informales y grandes proyectos urbanos en la Ciudad de Buenos Aires: el caso de la Villa 31}

\section{Informal settlements and large urban projects in the city of Buenos Aires: The case of Villa 31}

\author{
Melina Ons*
}

\section{Resumen}

La Villa 31-31 bis, o barrio Padre Carlos Mugica, es el asentamiento informal más antiguo y uno de los más grandes de la Ciudad de Buenos Aires. Se localiza cerca de los principales centros administrativos y de empleo de la ciudad, en un área con el precio del suelo más alto del distrito. El gobierno local está desarrollando alli un programa de reurbanización que fue sancionado por ley y dicta principalmente la instalación de servicios y la regularización dominial a través de hipotecas a cargo de los moradores. La intervención se da en paralelo a la recualificación y revalorización del entorno del asentamiento, de la mano de grandes proyectos urbanos públicos y privados. Se analiza en este artículo el contenido de la ley de reurbanización en el marco de la dinámica urbana y de las políticas de regulación de suelo en el cual se aplica.

Palabras clave: asentamientos informales; regularización dominial; grandes proyectos urbanos; regulación del suelo.

* Universidad Nacional de General Sarmiento, Instituto del Conurbano / Consejo Nacional de Investigaciones Científicas y Técnicas (Conicet). Dirección: Juan M. Gutiérrez 1150, CP B1613, Los Polvorines, Buenos Aires, Argentina. Correo: melinaons@gmail.com ORCID: https://orcid.org/0000-0001-8951-3897 


\begin{abstract}
The Villa 31-31 bis, or Padre Carlos Mugica neighborhood, is the oldest informal settlement and one of the largest in the City of Buenos Aires. It is located near the main administrative and employment centers of the city, in an area with the highest land prices in the district. The local government is developing an upgrading program that was sanctioned by law and, mainly, dictates the installation of public services and the regularization of tenure through mortgages paid by the residents. The intervention takes place as the settlements environment transits a requalification and revaluation process, hand in hand with large public and private urban projects. This article analyzes the content of the redevelopment law within the framework of urban dynamics and land regulation policies in which it is applied.

Keywords: informal settlements; regularization of tenure; large urban projects; urban regulation.
\end{abstract}

\title{
I. Introducción
}

En la mayor parte de los estudios urbanos ha cobrado fuerza el análisis sobre la espacialidad de las relaciones sociales y la forma en que la sociedad capitalista, en su fase globalizada, determina las características de la urbanización. Abramo (2008) afirma que, para cada etapa de la historicidad del capitalismo, corresponde un tipo de funcionalidad de las ciudades. El autor utiliza el concepto de "regímenes urbanos" para definir esas "formas particulares que asumen los procesos de producción y de apropiación de la espacialidad construida" (Abramo, 2008, p. 35). Según el autor, a principios de los años ochenta, luego de la crisis del régimen urbano fordista, comienza uno marcado por la flexibilización, la desregulación y la globalización de la economía. La instalación del neoliberalismo como modelo hegemónico a escala global tiene su traducción en la configuración de las ciudades. El Estado empieza a cederle lugar al mercado como coordinador de los usos del suelo y productor del espacio (Abramo, 2012) y abandona su rol como financiador de vivienda, equipamiento o servicios urbanos colectivos. 
Las regulaciones urbanísticas vigentes tienden a colocar el suelo disponible y el potencial para construir en función de las necesidades del mercado (Fernández Wagner, 2008). Esto habilita el surgimiento de operaciones inmobiliarias de gran escala que buscan desarrollar áreas deprimidas de la ciudad, pero bien ubicadas y con elevadas rentas potenciales, para revalorizarlas y obtener márgenes más amplios de ganancia (Smith, 2007; Cuenya, 2012). Cuenya define este tipo de emprendimientos como "grandes proyectos urbanos", que producen una revalorización del suelo mediante la rehabilitación de las áreas donde se asientan. Para su ejecución requieren la intervención del Estado y cambios en los mecanismos de gestión pública (Cuenya, 2012). Según la autora, estos emprendimientos encuentran su lógica en una dinámica urbana globalizada, ya que requieren la articulación con el capital financiero y están destinados a satisfacer la demanda de sectores de la economía trasnacional con alto poder adquisitivo. "Los grandes proyectos son nuevos espacios para nuevas formas de acumulación y de consumo" que pasan a formar parte de un mercado de bienes raíces de escala internacional (Cuenya, 2011, p. 188). El espacio se estructura para dar soporte material y simbólico a las actividades complejas de servicios, sobre todo tecnológicas y financieras -que comienzan a predominar por sobre la actividad manufacturera industrial-, y al alojamiento de la elite de las empresas globalizadas (Sassen, 1998).

En la Ciudad de Buenos Aires muchos de estos emprendimientos encontraron la posibilidad de asentarse en terrenos que estaban en desuso, como resultado de la privatización de empresas de servicios públicos, sobre todo ferrocarriles y puertos. Un ejemplo reciente es la refuncionalización y revalorización del barrio de Retiro y la parte norte de la rivera, de la mano de emprendimientos privados y obras públicas que, según el gobierno local, recibirán la mayor inversión de la historia del distrito. ${ }^{1}$ El plan, reactivado luego de varias décadas de debates y propuestas, tiene continuidades y semejanzas con el ejecutado durante los años noventa en la zona de Puerto Madero (Cuenya, 2012).

${ }^{1}$ https://www.buenosaires.gob.ar/noticias/rodriguez-larreta-macri-y-vidalanunciaron-el-inicio-de-las-obras-del-paseo-del-bajo 
En esa misma zona se emplaza la Villa 31-31 bis, uno de los asentamientos más grandes y emblemáticos de la ciudad, que es objeto de un programa de reurbanización iniciado en el año 2016, y cuyo principal financiamiento proviene del Banco Interamericano de Desarrollo (BID) y el Banco Mundial. Las características principales de esta política se han plasmado en una ley sancionada en 2018, que abordaremos en este artículo.

Los asentamientos informales también son resultado de la dinámica urbana que describimos al comienzo. Como afirma Smolka (2002), este tipo de urbanizaciones encuentran sus causas en la pobreza, pero también en "la falta de programas habitacionales, la disminución de inversiones públicas en equipamientos urbanos y servicios, y el propio vaciamiento del planeamiento urbano" (Smolka, 2002, p. 5), que redundan en una escasa oferta de suelo urbanizado para amplios sectores de la población. Ante la imposibilidad de acceder a la ciudad por vía del mercado o del Estado, surge la vía de la necesidad que promovió el desarrollo de la informalidad en América Latina como norma y no como excepción (Abramo, 2012; Fernandes, 2003).

Estudios precedentes sostienen que, dado el carácter escaso del suelo, la existencia de asentamientos en áreas servidas y con alto valor potencial puede convertirse en un obstáculo para la reproducción del capital, ya sea porque impide que los terrenos ocupados se incorporen a la dinámica de valorización, o porque la marginalidad se conforma como una externalidad negativa para los nuevos desarrollos. Se pone en tensión un entramado de actores con intereses contrapuestos, en el que la existencia de territorios ocupados por los asentamientos informales puede dar lugar a fuertes disputas por su posesión (Cravino, 2009). La intervención del Estado adquiere entonces un rol central. Las distintas formas de acción pública sobre los asentamientos informales tienen diferentes visiones sobre lo público y lo privado, y sobre cómo se distribuye o redistribuye la riqueza que surge del proceso urbanizador (Acosta, 2019). Del mismo modo, pueden tener efectos diversos en amortiguar o incrementar el impacto social y espacial de la pobreza (Wacquant, 2007).

El objetivo de este artículo es analizar el programa de reurbanización de la Villa 31-31 bis en el marco de la renovación de su 
entorno, teniendo en cuenta la dinámica urbana de manera integral. Comenzaremos caracterizando la Villa 31-31 bis, luego los emprendimientos inmobiliarios en curso en la zona y el programa de regularización para el asentamiento. Concluiremos con algunas reflexiones sobre los posibles resultados de este último, que, al ser un fenómeno en desarrollo, sólo pueden tener carácter de hipótesis.

\section{La historia de la Villa 31-31 bis: entre la erradicación y la reurbanización}

La Villa 31-31 bis está ubicada en la zona de Retiro, cerca de los principales centros administrativos y de empleo de la Ciudad de Buenos Aires. Es uno de sus asentamientos informales más grandes y emblemáticos; residen allí cerca de 40 mil personas $^{2}$ y está conformado por cinco barrios internos. Es también la villa más antigua de la ciudad: sus primeros habitantes se instalaron en la década de 1930. Su ubicación estratégica y el valor potencial de sus terrenos dieron lugar a un variado derrotero en relación a la estabilidad de los moradores en las tierras ocupadas y a posturas oficiales que oscilaron entre la erradicación y la radicación (Cravino, 2009).

La última dictadura militar argentina (1976-1983) intentó desalojar el barrio de manera forzosa, destruyendo la mayoría de las viviendas. Pero 43 familias lograron resistir el desalojo y, recuperada la democracia, el asentamiento volvió a crecer hasta la actualidad. En 2007, cuando Mauricio Macri asumió su primer mandato como jefe de gobierno de la ciudad, declaró que la Villa debía ser erradicada para destinar los terrenos al puerto. ${ }^{3}$ Pero la organización de los ve-

${ }^{2}$ El último censo poblacional realizado que fue publicado por el Gobierno de la Ciudad, en el año 2009, informa que residen allí 26403 personas. https://www. estadisticaciudad.gob.ar/eyc/wp-content/uploads/2015/04/villa_31_y_31_ bis_2009_abril.pdf. Pero el barrio ha crecido mucho desde entonces, y la información más reciente determina que este número asciende a 40 mil personas. https://www. clarin.com/ciudades/haran-mercado-gastronomico-villa-31-intentar-atraer-vecinos -toda-ciudad_0_r1_mpXiIX.html

${ }^{3}$ http://www.lanacion.com.ar/919873-no-voy-a-permitir-que-haya-cortes-decalles 
cinos y un consenso general contrario a las erradicaciones, lograron revertir la definición oficial y, junto con académicos, movimientos sociales, políticos y legisladores, elaboraron una ley de urbanización que fue aprobada en el año 2009 (núm. 3 343, CABA).

La mencionada ley suspendía los desalojos, determinaba los límites del polígono ocupado y ordenaba la reurbanización, cuyos detalles deberían ser definidos en una norma complementaria posterior. Para elaborar esta última se conformó una mesa multidisciplinaria y participativa que, en el año 2011, presentó un proyecto con detalles técnicos urbanísticos. El mismo debía ser aprobado por el parlamento local, pero la postura del gobierno ya no se mostraba favorable a la urbanización, y la correlación de fuerzas de la organización vecinal ya no era la misma que años atrás. El plan no se aprobó, y en los años siguientes el Estado sólo realizó intervenciones menores en el barrio. Tampoco se desarrollaron en ese período políticas de gran envergadura en otros asentamientos de la ciudad. ${ }^{4}$

En el año 2015 Mauricio Macri asumió la presidencia de la nación, y como jefe de gobierno de la ciudad lo hizo Horacio Rodríguez Larreta, un miembro de su partido que había sido su vicejefe en mandatos anteriores. Este último anunció que llevaría adelante la reurbanización de las principales villas del distrito. Como hemos analizado en trabajos anteriores (Ons, 2018), este cambio en la concepción gubernamental, de la erradicación en 2007 a la regularización casi diez años después, tuvo dos motivos principales. Por un lado, un hecho casi inédito en la historia de la autonomía porteña: el gobierno de la ciudad y el de la nación pasaron a estar conducidos por el mismo partido. Además de fortaleza política, esto le otorgó a la ciudad la posibilidad de disponer de recursos y tierras nacionales con las que hasta entonces no contaba-como aquellas ocupadas por la Villa 31 y otras que se destinaron a proyectos privados-. El segundo factor que acompaña la política de reurbanización es una tendencia regional a reconocer los asentamientos informales e intervenir en ellos. En términos de Fernandes (2003):

${ }^{4}$ Acerca del debate parlamentario y la acción pública en torno a la urbanización de la Villa 31-31 bis en el período 2007-2015, consúltese Ons, 2018, en https:// dialnet.unirioja.es/servlet/articulo?codigo $=6566127$ 
Dada la creciente movilización social de estas comunidades, que claman por el reconocimiento de sus derechos y por el mejoramiento de las áreas, las políticas públicas también han evolucionado en el sentido de reconocer en alguna medida su derecho de tener un lugar en el espacio de la ciudad [Fernandes, 2003, p. 10].

En este marco, en su discurso de asunción en diciembre de 2015, el nuevo jefe de gobierno, Horacio Rodríguez Larreta, anunció la reurbanización de cinco villas porteñas. La mayoría serían realizadas por el Instituto de Vivienda de la Ciudad, pero, dada su magnitud e importancia, se creó una subsecretaría especial para la Villa 31-31 bis. Tal organismo lleva el nombre de Subsecretaría de Integración Social y Urbana (SISU) y comenzó a intervenir en el barrio con financiamiento del BID y del Banco Mundial, mientras se elaboraba un nuevo proyecto de ley de reurbanización. El objetivo era presentar una norma en el parlamento local que complementara la existente. Esto ocurrió y en 2018 la ley se aprobó en la Legislatura (Ley CABA núm. 6129). A los detalles de la norma nos referiremos en apartados posteriores.

\section{La ubicación de la Villa en la Ciudad de Buenos Aires y los grandes proyectos urbanos en Retiro}

Hasta la crisis política y económica del año 2001 en Argentina, la Villa 31 era la única ubicada en el norte del territorio de la ciudad, el cual tiene mejores indicadores socioeconómicos, sanitarios, habitacionales y educativos que la franja sur (donde se encuentra la mayor parte de los asentamientos). Es lindera a los barrios de mayor poder adquisitivo - un área de tradicional importancia comercial y residencial-, y está separada de éstos por las vías del ferrocarril, tal como se muestra en la Fotografía 1.

El área que ocupa la Villa presenta los mayores valores de incidencia del suelo de la ciudad, como puede observarse en el Mapa 1. ${ }^{5}$

${ }^{5}$ El Mapa 1 expresa los valores en las llamadas unidades de valor adquisitivo (UVA). Ésta es una unidad creada por el Gobierno Nacional en 2018 para ajustar una línea de créditos para vivienda. El valor de las UVA las define diariamente el Banco 
Como se aprecia, el valor del polígono a reurbanizar es relativamente más bajo que el de su entorno.

\section{Fotografía 1}

El barrio de Retiro y San Nicolás, las vías del ferrocarril y la Villa 31

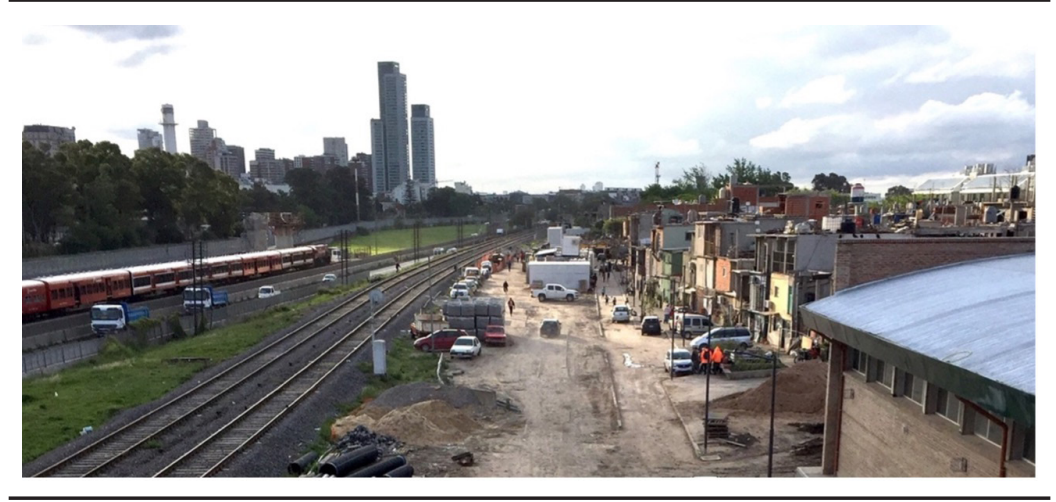

Fuente: Archivo propio.

Pese a su localización privilegiada y al valor potencial del suelo, el área específica que ocupa estuvo relativamente desconectada de la dinámica urbana: una zona anteriormente deprimida con áreas vinculadas a la actividad portuaria, del ferrocarril o de la empresa nacional de hidrocarburos que se encontraban en desuso. Actualmente, en paralelo a la reurbanización, se están desarrollando grandes proyectos urbanos públicos y privados que operan en conjunto con la urbanización del barrio, en un proceso de refuncionalización de la zona.

En este marco, en el año 2016 comenzaron algunas obras de infraestructura impulsadas por el Gobierno de la Ciudad de Buenos Aires (GCBA), sobre todo viales y de transporte.

Los principales proyectos impulsados por el poder público son los siguientes:

Central de la República Argentina, y es ajustado según el coeficiente inflacionario nacional. Por poner una referencia, el 21 de octubre de 2019 (al cierre de este artículo), el valor de esta unidad era US\$ 0.7 . 
- Construcción del Paseo del Bajo (Ley núm. 6131, dic., 2016): una autovía recientemente inaugurada de $7 \mathrm{~km}$ de largo que une los ingresos norte y sur de la ciudad, y que tuvo un costo estimado de US\$ 700 millones.

- Nueva traza de la Autopista Illia (Ley núm. 5733, dic., 2016): con un presupuesto inicial de US\$ 90 millones, el proyecto consiste en modificar el recorrido existente, pero se encuentra actualmente suspendido.

- Concesión de la estación central de autobuses para su reconversión en un complejo hotelero-turístico a cargo de empresas privadas (sancionada por la Ley núm. 6152 en marzo de 2019, actualmente en licitación).

- Modernización de las estaciones del ferrocarril Mitre (año 2017) y San Martín (en desarrollo).

\section{Mapa 1}

Valores de incidencia del suelo por manzana.

Ciudad de Buenos Aires, 2019

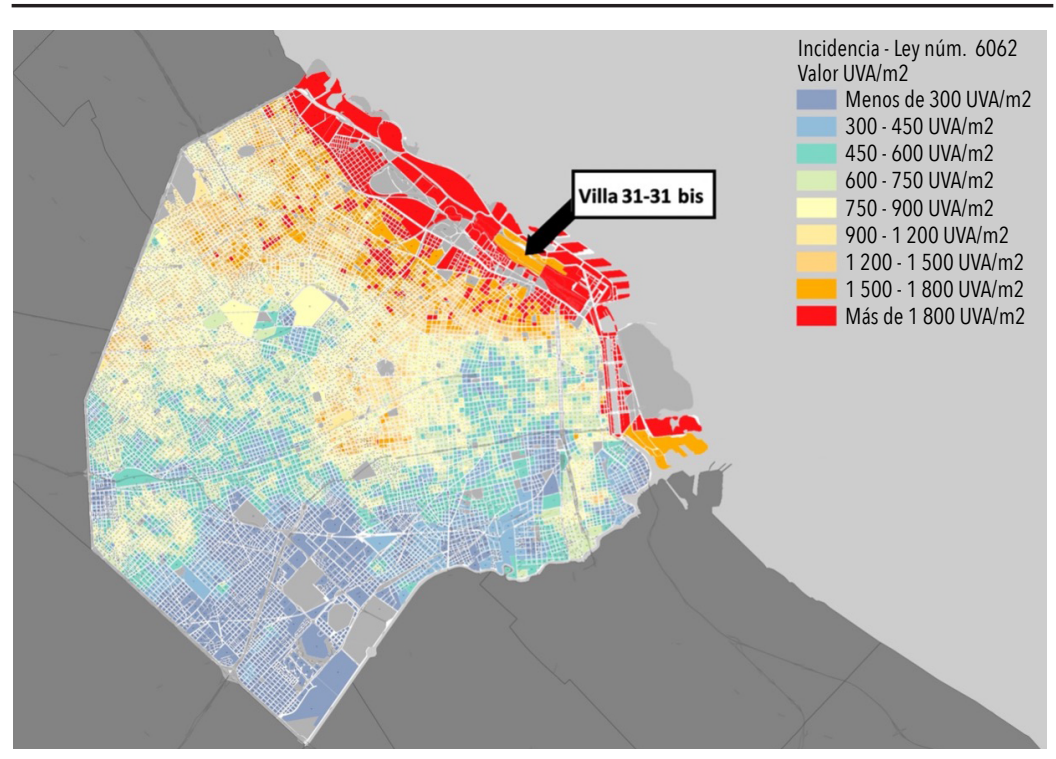

Fuente: Gobierno de la Ciudad de Buenos Aires. 
La obra principal, por su magnitud y la movilización de recursos que implicó, es la del Paseo del Bajo, financiado en mayor parte con lo recaudado por la subasta de terrenos públicos. ${ }^{6}$ En su último tramo, el Paseo del Bajo bordea la Villa 31, en la parte en la que se está construyendo un complejo de viviendas nuevas para las relocalizaciones que fueren necesarias, tal como se observa en la Fotografía 2.

\section{Fotografía 2}

Viviendas nuevas dentro del barrio y Paseo del Bajo en construcción, Villa 31, año 2018

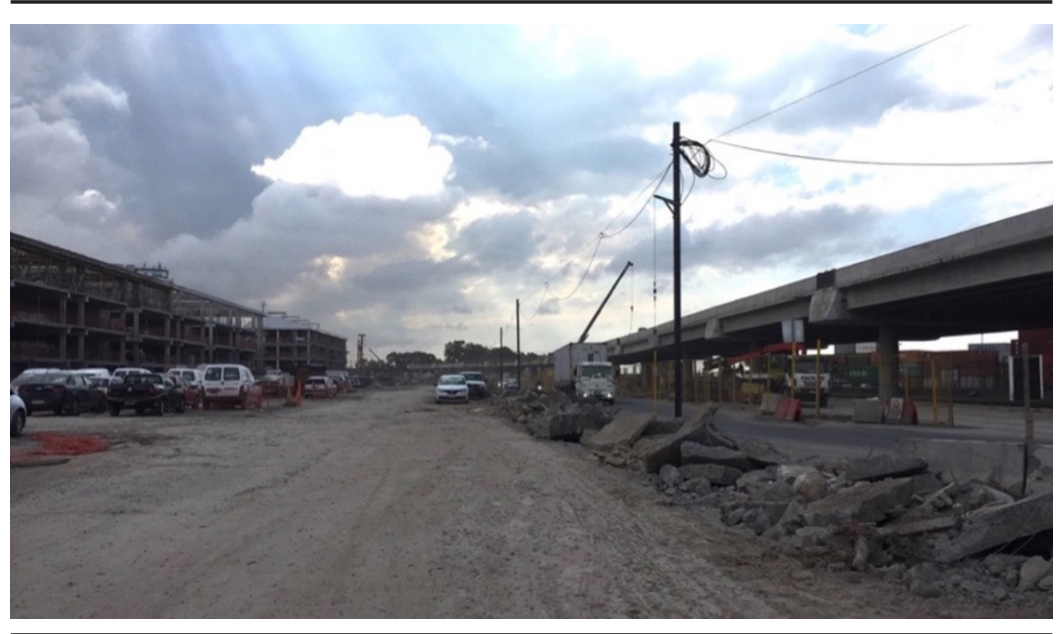

Fuente: Archivo propio.

Aunque no existe al momento un censo oficial y público de población de la Villa, la información existente determina que el grueso de las relocalizaciones a realizarse, de acuerdo con el programa de reurbanización, corresponde a las familias que viven debajo de la autopista Illia. Esta última vía actualmente atraviesa el barrio y es la principal conexión rápida entre el centro de la ciudad y los distritos del norte del conurbano. El citado plan del gobierno busca clausurar su recorrido actual y construir uno nuevo a pocos metros, bordeando

${ }^{6}$ https://www.clarin.com/ciudades/nacion-cede-tierras-ciudad-regularizarviviendas-villas-financiar-obras_0_DwpWj47pu.html 
el barrio. El objetivo es que, cuando la vieja traza quede en desuso, se construya en la antigua un parque lineal en altura con comercios y espacio verde. La reforma se encuentra suspendida, ${ }^{7}$ pero la urbanización incluye la reubicación de todas las viviendas que se colocaron debajo durante la autoconstrucción del asentamiento, como las que se observan en la Fotografía 3.

\section{Fotografía 3}

Viviendas autoconstruidas debajo de la autopista Illia

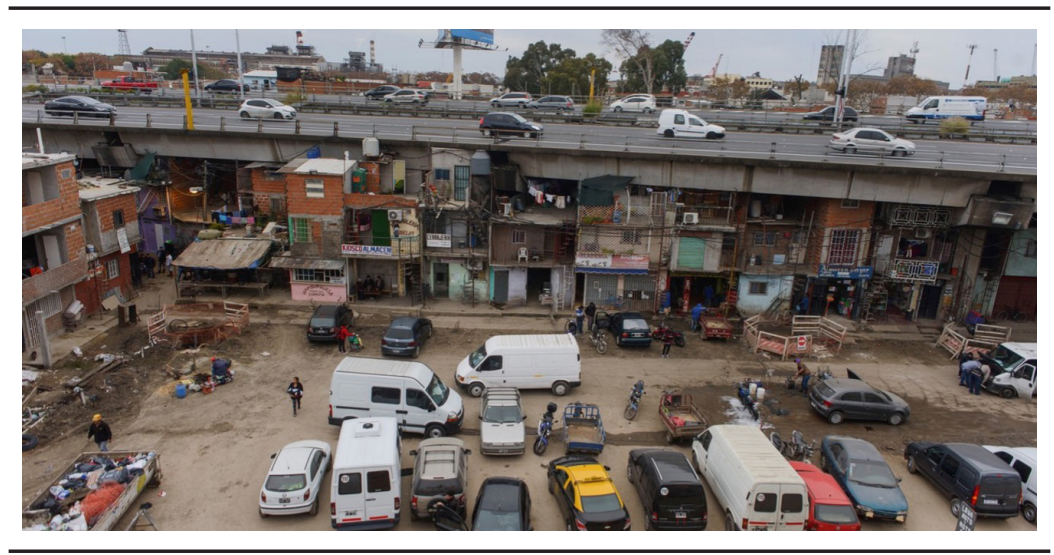

Fuente: Diario Clarín.

En conjunto con los proyectos de infraestructura impulsados por el poder público antes mencionados, se están desarrollando otros emprendimientos a cargo de empresas privadas. Los mismos cuentan con la inversión pública como condición de posibilidad para generar ganancias. Así lo reconocía uno de los voceros del fondo común de inversión que lleva adelante el distrito Quartier Retiro, uno de los principales emprendimientos de la zona. En la reunión de presentación del proyecto, realizada ante posibles inversores en el año 2017, el expositor planteaba lo siguiente:

${ }^{7}$ Hasta el momento este proyecto se encuentra suspendido por falta de recursos. https:/www.perfil.com/noticias/sociedad/frenan-hasta-2019-las-obras-para-moverla-autopista-illia.phtml 
Van a suceder, en el área que les muestro acá, una serie de inversiones públicas que van a cambiar la fisionomía del lugar. La invitación del fondo [de inversión] es a formar parte del diferencial que se genera en el valor de la tierra, en una tierra que se reposiciona en valor porque se reconvierte la zona [...] En este caso, vemos un cambio de área con el turbo que nos pone la inversión pública, que normalmente no tenía. ${ }^{8}$

Además de la inversión pública, los proyectos en desarrollo contaron con la habilitación del Estado a través de normas aprobadas en la legislatura porteña. A continuación, se detallan los principales emprendimientos privados y sus normas habilitatorias:

- Catalinas Norte II: privatización y rezonificación de un terreno de siete lotes que pertenecía a la Agencia de Administración de Bienes del Estado (AABE); fue transferido a la ciudad y adquirido en subasta por empresas desarrolladoras. Además de la enajenación, la Legislatura aprobó allí el desarrollo de torres de residencias y oficinas (Ley núm. 5796, mar., 2017).

- Distrito Quartier Retiro: barrio de $7800 \mathrm{~m}^{2}$ con tres edificios para viviendas, oficinas y áreas verdes. Anteriormente funcionaba un hospital de los trabajadores ferroviarios. El nuevo desarrollo se habilitó gracias a la rezonificación del terreno (Resolución núm. 193, mayo, 2017).

- Edificio puente BID: el GCBA cede de manera gratuita por 100 años un terreno para la construcción de un edificio de oficinas del organismo de financiamiento (Ley núm. 6130, dic., 2018).

En el Mapa 2 se observan los principales desarrollos públicos y privados en torno a la Villa.

A esta lista se suma la privatización de los terrenos que ocupa el barrio y otros en su entorno, por parte de la AABE. Además de los que serán privatizados mediante subastas, se incluyeron los que ocupan los habitantes del asentamiento para la posterior titularización a su favor. Existen importantes incertidumbres entre estos últimos

${ }^{8} \mathrm{La}$ presentación completa puede consultarse en https://www.youtube.com/ watch?v=PPdcDHTG-MY\&list=PLRUO32M1CvrQYpSo2e_xfZHGU2jZAotrV \&index $=4$ 
acerca de cuáles serán las tierras que corresponderán al polígono de reurbanización y cuáles serán compradas por otros agentes del mercado inmobiliario.

\section{Mapa 2}

Grandes proyectos urbanos en desarrollo en el entorno de la Villa 31-31 bis

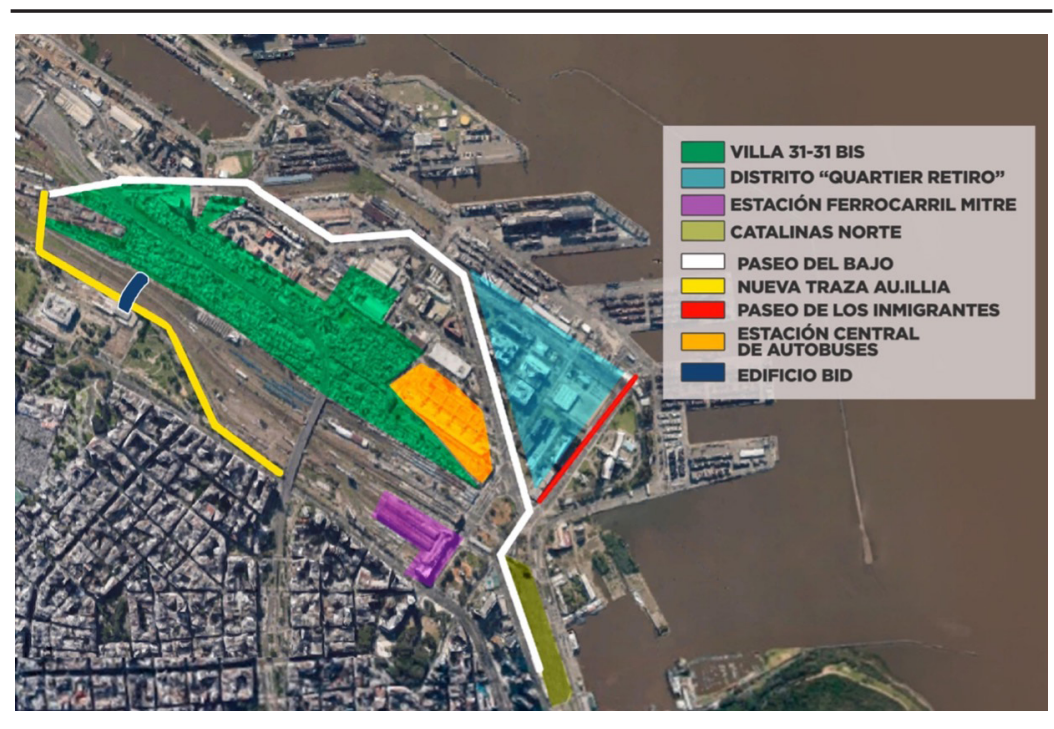

Fuente: Elaboración propia.

En resumen, el proyecto de reurbanización de la Villa 31-31 bis se desarrolla en un contexto de recualificación del entorno, y consideramos necesario analizarlo en ese marco. El Gobierno de la Ciudad sostiene que espera poder generar en la zona una "mixtura social" que incluya los grandes desarrollos y la reurbanización del barrio. Al respecto, en una de las entrevistas realizadas a un coordinador de la SISU, el mismo declaró:

Hoy el barrio necesita servicios, necesita comercios, necesita generar mixtura social. A mí me parece que la mixtura social, como nosotros la estamos entendiendo, tiene que ver con no dejar que el mercado se haga cargo, sino que sea el Estado el que genere esa mixtura social. El 
Estado y el mercado son las dos grandes fuerzas que, de alguna manera, configuran la espacialidad urbana. ${ }^{9}$

\section{Ley de urbanización núm. 6129 del barrio Padre Carlos Mugica (Villa 31-31 bis)}

Como se mencionó anteriormente, en el año 2015 el gobierno local anunció la reurbanización. Luego de eso, inició algunas intervenciones en 2016 y convocó a una mesa técnica para elaborar el proyecto urbanístico que se aplicaría en la Villa 31-31 bis o barrio Padre Carlos Mugica. Dicha mesa estaba compuesta por representantes de la SISU, del asentamiento, de los distintos poderes del Estado local, y de organizaciones de la sociedad civil. El resultado fue una política pública con formato de ley que se aprobó en la legislatura porteña en 2018 (Ley CABA núm. 6 129). La misma establece nuevos usos del suelo, apertura de calles y lineamientos para la regularización dominial mediante hipotecas a cargo de los moradores.

La norma no contó con el mismo apoyo con el que se aprobó la primera en 2009. La mayoría de los legisladores de la oposición no votó su aprobación y existieron muchas disidencias con el articulado final por parte de los propios miembros de la mesa técnica y de una porción de los habitantes. Las principales críticas se asociaron a la poca participación de la comunidad en su elaboración y a la falta de definición de componentes centrales, como el precio que los beneficiarios pagarán por las viviendas en las que habitan, o por las nuevas en caso de ser relocalizados. Esto generó fuertes enfrentamientos de los habitantes entre sí y de éstos con los funcionarios y legisladores oficialistas, lo cual da cuenta de la complejidad de actores, intereses, políticas y espacios que configuran el orden urbano.

A continuación, enumeraremos los puntos centrales de la normativa que interesan a los fines de este trabajo.

\footnotetext{
${ }^{9}$ Entrevista realizada en el marco de esta investigación, en octubre de 2018.
} 


\title{
IV.1. Regularización dominial
}

\author{
Pago de parcelas
}

El artículo 58 de la Ley establece:

En el marco del proceso de reurbanización e integración social, será responsabilidad de todos/as los/as beneficiarios/as contribuir, en la medida de sus capacidades y sin perjuicio de su subsistencia, con el pago proporcional a la adjudicación de las parcelas y/o unidades funcionales. En ningún caso la incapacidad de pago vulnerará los derechos reconocidos en la presente Ley a favor de los/as beneficiarios/as [Ley CABA núm. 6129$].{ }^{10}$

Complementariamente, su artículo 52 dicta:

Posterior a la primera escrituración, los/as adjudicatarios/as podrán disponer de su inmueble siempre que:

a) Cancelen el valor total de la hipoteca constituida sobre el inmueble.

b) En caso de ser beneficiarios/as de una solución única y definitiva según los términos referidos en el art. 30, demuestren ante la Autoridad de Aplicación contar con otra solución habitacional definitiva, acorde al grupo familiar [Ley CABA núm. 6129].

Tal como indican los artículos citados, los pobladores acceden a los inmuebles que han construido, o a aquellos a los que serán relocalizados, abonando un valor mediante hipoteca al GCBA. La ley no fija el precio ni los criterios generales que se utilizarán para establecerlo. Al cierre de este artículo tampoco se había definido este monto en ninguna disposición complementaria. Esto ha generado una gran incertidumbre entre los habitantes que forman parte de los estratos más bajos de la población y que, en su mayoría, poseen empleos informales con ingresos inestables.

La norma tampoco expresa que se vaya a descontar del monto de la hipoteca el valor de la vivienda autoconstruida, ni se explici-

${ }^{10} \mathrm{http} / / /$ api-restboletinoficial.buenosaires.gob.ar/download/1276128 
tan subsidios para la mejora de las construcciones existentes que no serán realojadas. Sí determina que las viviendas deberán tener conexión a los servicios públicos para la escrituración y fija una política de financiamiento con cuotas fijas, una tasa del $4 \%$ y en un plazo extendible entre 36 y 40 años según la capacidad de pago (arts. 27 y 60 , respectivamente).

La forma de financiar este tipo de urbanizaciones ha sido objeto de importantes discusiones, ya que se trata de intervenciones muy costosas. El pago total de los predios a cargo de los habitantes tiene pocos antecedentes en procedimientos de regularización en América Latina. En otras experiencias en la región, el intento de trasladar los costos a los residentes ha tenido fuertes resistencias (Fernandes, 2011) y no se registran casos en los que éstos hayan tenido que abonar el precio pleno. ${ }^{11} \mathrm{Si}$ bien son experiencias diversas por el origen de la propiedad del suelo ${ }^{12}$ y las características de las políticas aplicadas, se pueden citar algunos ejemplos. En el caso de la regularización de la tenencia de la tierra en México, primero bajo el Programa de Regularización de la Comisión de Regularización de la Tenencia de la Tierra (Corett) y luego bajo el Programa de Certificación de Derechos Ejidales y Titulación de Solares (Procede), implementado después de la reforma a la Ley Agraria en 1992, se otorgaron títulos de propiedad a cambio de bajos costos administrativos para

${ }^{11}$ En la Ciudad de Buenos Aires, la reurbanización de la Villa 20, que se está desarrollando en paralelo a la de la Villa 31-31 bis, también tiene un régimen de pago por la vivienda nueva, pero se descuenta de ella el valor de la vivienda autoconstruida, y el Instituto de Vivienda de la Ciudad subsidia las mejoras necesarias para garantizar la habitabilidad. Además, existe un mecanismo establecido previamente para el cálculo del precio de cada unidad.

${ }^{12}$ En el caso mexicano, la tierra era de propiedad ejidal y tuvo que pasar por un proceso de expropiación; mientras que en los casos de Perú y del ProMeBa eran propiedad estatal. También hubo diferencias en cuanto al rol que cada uno le daba a la regularización. En los casos peruano y mexicano, la solución que encontró el Estado, ante la imposibilidad de proveer viviendas, fue la entrega de los títulos de las existentes. En Brasil, en cambio, se implementó una serie de medidas integrales desde la perspectiva del derecho social a una vivienda digna, promovido por el Programa de las Naciones Unidas sobre los Derechos de la Vivienda (Fernandes, 2011). El ProMeBa también contempla la urbanización como un proceso de mejoramiento integral de las condiciones de vida de las familias. 
los pobladores y también de forma gratuita para los ejidatarios (Ibarra 2013; Salazar, 2020); en las favelas regularizadas de Brasil se ha convocado a los habitantes al pago en especie, la autoconstrucción o la ayuda mutua; y en la legalización de asentamientos informales en Perú se entregaron también títulos de propiedad con bajos costos administrativos para los ocupantes (Fernandes, 2011). Cabe retener que también en Argentina, en casos como el Programa de Mejoramiento en Barrios (ProMeBA, 1997 y 2007), se establece por ley la existencia de consultores legales que trabajan en el territorio con los pobladores a fin de ajustar el precio y la forma de pago de acuerdo con la capacidad económica de las familias (Almansi, 2009).

Recargar a los pobres con obligaciones financieras puede constituir una penalidad, más que un aporte a la resolución del problema de la marginalidad. Estas obligaciones podrían condicionar de forma significativa la estabilidad de los habitantes en el lugar en el que residen y reforzar el conjunto de causas que generaron la informalidad (Fernandes, 2003). Los pagos por infraestructura y mejoras deben ser compatibles con la capacidad de pago de cada actor y, en el caso de los asentamientos informales, deben responder a los mismos principios que se aplican en las áreas formales que fueron beneficiadas con la intervención pública (Fernandes 2011). Las cargas económicas que se generen sobre la población vulnerable podrían ser desproporcionadas en relación a las que pesan sobre otros actores económicos que se ubican o pretenden ubicarse en la zona. Los emprendimientos privados que allí se ubican -en la zona aledaña a la Villa, por ejemplo-, se verán beneficiados por el aumento del precio del suelo que se producirá por la intervención del Estado, dado que las acciones del poder público como la reurbanización de la Villa 31 , las medidas administrativas para autorizar desarrollos o la inversión en mejorar la infraestructura vial, generarán importantes márgenes de ganancia que no son producto de la acción privada individual, sino de operaciones urbanas con inversión pública.

El aumento del valor puede ser considerado como un "esfuerzo de la comunidad", susceptible de ser recompensado (Smolka y Furtado, 2001). La regulación urbanística vigente en la Ciudad de Buenos Aires no exige a estos propietarios o desarrolladores que realicen aportes para compensar la valorización que surja de la acción del Es- 
tado y no de las inversiones privadas. Diversos autores sostienen que el desarrollo de infraestructura común o la construcción de vivienda social podrían financiarse con lo que se llama "recuperación de plusvalías", entendidas como las ganancias extraordinarias generadas por acciones gubernamentales, ajenas a los propietarios individuales. Se hace pertinente aquí una pregunta que introduce Cuenya (2011):

¿Las actuaciones públicas municipales en grandes proyectos urbanos implican una subvención al capital inmobiliario y a consumidores ricos a expensas de los recursos públicos, o abren la oportunidad para que el sector público capture los beneficios de las inversiones y las distribuya para mejorar las condiciones de vida de los sectores sociales en desventaja? [Cuenya, 2011, p. 15].

Sostenemos aquí que, con instrumentos de recuperación de plusvalías que repartan las cargas y los beneficios de la ciudad de manera más equitativa, podrían generarse ingresos al erario público como para financiar la urbanización de la Villa, reduciendo el endeudamiento con organismos internacionales y aliviando el peso sobre los ingresos de los pobres.

\section{Tamaño del espacio a regularizar}

En su artículo 51, la norma determina que los habitantes pueden acceder a la vivienda autoconstruida y ceder el espacio potencialmente construible al Gobierno de la Ciudad. Así manda dicho artículo:

Art. 51. El Gobierno de la Ciudad de Buenos Aires podrá acceder a la capacidad constructiva remanente, de conformidad a la normativa urbanística vigente al momento, lo cual se materializará en la primera escritura traslativa del dominio mediante una cesión de derechos sobre los metros edificables [Ley CABA núm. 6129].

De este modo, el espacio aéreo no construido, según la zonificación establecida en cada área del polígono, queda a disposición del Estado. Si bien no se establece cuál será el uso que se les dará, el citado coordinador del programa afirmaba que: 
Hay que generar aperturas de patios, iluminación, etc. Y para todo ese tipo de reconfiguración, lo mismo para una apertura de calle, se puede realizar una intervención sobre el espacio aéreo de un vecino que no haya formalizado. ${ }^{13}$

Este tipo de control se asemeja a las herramientas urbanísticas que se aplican en otras partes de América Latina para separar los derechos de propiedad de los de construcción. Podría tratarse, por ejemplo, de una transferencia de potencialidades, instrumento que se utiliza principalmente en Colombia y Brasil, que supone la compra-venta de capacidad edificatoria de unos inmuebles a otros, según autorice la normativa urbana, para el cumplimiento de objetivos locales (Maldonado, 2013). También puede referirse al Otorgamiento de Derechos de Construcción, que en Brasil se aplica de manera similar, pero con fines distintos a lo establecido en la ley que aquí se analiza. En la experiencia brasileña, el Estado puede ser dueño de los derechos de construir por encima de determinado nivel y venderlos en el mercado, para financiar con los recursos recaudados el desarrollo de otras zonas de la ciudad que lo requieran. Esto "supone la idea de que el derecho a construir no se deriva del derecho de propiedad, sino de actos de la administración" (Azuela, 2016, p. 27).

Sin embargo, no hay instrumentos de esas características vigentes en otras partes de la Ciudad de Buenos Aires, por lo que su aplicación significaría un cambio importante en la concepción acerca de los aportes que los propietarios de inmuebles con capacidad constructiva mayor a la existente pueden realizar al bien común. Repensar la propiedad, en lugar de como un derecho absoluto, como una multiplicidad de derechos y obligaciones, permitiría generar un balance entre el beneficio social e individual, además de permitir mejorar las finanzas públicas. Pero si se aplica sólo para los habitantes de la Villa 31 y no como norma al conjunto de la ciudad, en el marco de un plan urbano general, pierde su potencial como instrumento redistributivo de planificación y se convierte en una limitación exclusiva para los futuros propietarios regularizados.

${ }^{13} \mathrm{https}$ //www.youtube.com/watch?v=PPdcDHTG-MY\&list=PLRUO32M1 CvrQYpSo2e_xfZHGU2jZAotrV\&index $=4$ 


\section{IV.2. Régimen de propiedad}

Una vez adquirido el inmueble por vía de la hipoteca, el programa establece la forma en la que éste puede ser enajenado. Explicita también la forma de adquirirlo que tendrían los compradores no residentes. Así se describe en el articulado:

Art. 52. Posterior a la primera escrituración, los/as adjudicatarios/as podrán disponer de su inmueble siempre que:

a) Cancelen el valor total de la hipoteca constituida sobre el inmueble.

b) $[\ldots]$ demuestren ante la Autoridad de Aplicación contar con otra solución habitacional definitiva, acorde al grupo familiar.

Art. 53.- En el marco del artículo precedente, cuando el comprador no acredite residencia mayor a dos (2) años en el Barrio Padre Carlos Mugica, deberá abonar tres (3) veces el valor de la hipoteca establecido al momento de la primera escrituración y valuada en unidades de valor adquisitivo (UVA) [Ley CBA núm. 6 129]. ${ }^{14}$

Es decir, un adjudicatario de la propiedad, una vez saldada la hipoteca, puede luego venderla a un comprador que no vive en el barrio, siempre que éste tenga capacidad de pagar tres veces su valor como penalidad. Esto podría tomarse como un desaliento a la compra de lotes por parte de actores externos a la Villa, pero también puede ser entendido como una manera de fijar las reglas para su enajenación, dependiendo del valor que se otorgue en un futuro a las hipotecas y de la transformación que puedan generar los desarrollos de la zona. Es de destacar que el proyecto no establece límites a la sumatoria de parcelas a nombre de un mismo comprador o al anexamiento de lotes.

Si no se acompaña con mecanismos de protección a la propiedad regularizada, que garanticen la permanencia de los actuales habitantes, la medida descrita puede tener como consecuencia la mercantilización de los lotes formalizados. En un paralelismo a lo que describe Salazar (2014) para el caso del nuevo marco jurídico-institucional en México a partir de la reforma de la Ley Agraria

${ }^{14}$ http://api-restboletinoficial.buenosaires.gob.ar/download/1276128 
de 1992, la habilitación en la Villa 31 puede permitir la entrada de nuevos actores inmobiliarios con mayor poder económico pero que actúan como "pares jurídicos" de la población vulnerable (Salazar, 2014, p. 87). La autora señala que, a diferencia de los pobladores de tierras no regularizadas, los promotores inmobiliarios "cuentan con todos los recursos económicos y sociales disponibles a su favor para apropiarse de la tierra y con la sapiencia jurídica para manejar el negocio inmobiliario y obtener plusvalías de éste" (Morales, 2002, en Salazar, 2020). La igualdad ante la ley no revierte la desigualdad entre los agentes que interactuarían en el mercado que se habilita con la formalización.

La falta de información y precisiones acerca de la forma de acceder a la vivienda actúa como un agravante. Como señala Abramo (2008), "el juego de la disputa mercantil exige que los agentes expresen sus actos basados en anticipaciones y, por tanto, procuren alcanzar el máximo de información posible" (Abramo 2008, p. 156). En este intercambio, la información pasa a ser un bien fundamental, y la asimetría en el acceso a la misma puede generar perjuicios para los actores en desventaja. Los habitantes del barrio, a diferencia de los desarrolladores inmobiliarios, han permanecido fuera del mercado formal de tierras, generando prácticas específicas de la informalidad. La transición a la formalidad requiere provisión de información y formación que les otorguen fortalezas dentro del marco jurídico en el cual se insertarán (Salazar, 2020).

\section{IV.3. Cambios en la dinámica económica y espacial}

Según el Código de Planeamiento Urbano anterior a la sanción de la Ley, los terrenos que ocupa la Villa 31-31 bis no tenían usos compatibles con las actividades necesarias para el barrio reurbanizado. Por ello, la norma establece la rezonificación del polígono. Sanciona un cuadro de usos nuevo que, además de habilitar el residencial, aumenta el uso comercial y alienta el desarrollo de las parcelas ubicadas sobre las principales vías de acceso, estableciendo allí una capacidad constructiva mayor y un tamaño diferente de las parcelas respecto de las áreas internas. 
En su Anexo núm. III, la Ley sanciona que "Se deberá considerar para todo el polígono un área máxima destinada a uso comercial del 20\% de total del área construible" (Ley CABA núm. 6129. http:// api-restboletinoficial.buenosaires.gob.ar/download/1276128). Luego define el carácter urbanístico de las parcelas exteriores: "Subzona 1c. Carácter urbanístico: áreas residenciales de media densidad, de potencial y preferente desarrollo por su ubicación estratégica". Hace referencia a las parcelas frentistas a corredores, vías principales y a espacios públicos de gran escala.

En el Mapa 3 se observan los niveles de construcción autorizados por la nueva zonificación. Las partes más oscuras responden a alturas más altas de edificación y coinciden con las zonas exteriores del barrio.

\section{Mapa 3}

\section{Rezonificación de la Villa 31}

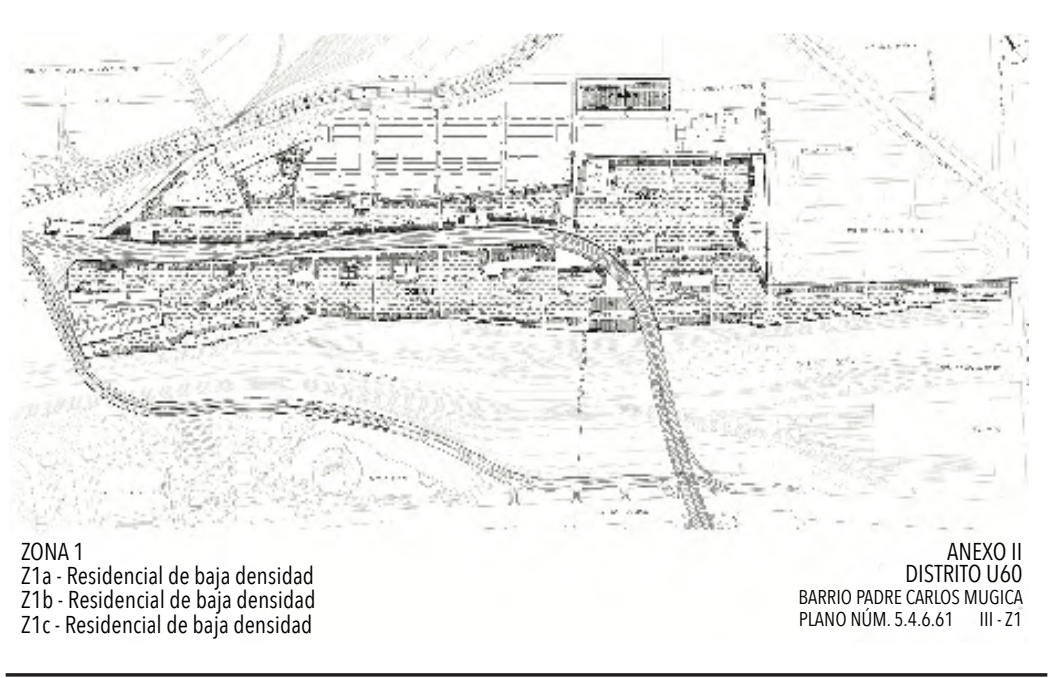

Fuente: Ley GCBA6129/18, Anexo I.

Esta nueva reglamentación no sólo podría modificar la configuración espacial y la dinámica comercial interna, caracterizada por una economía de subsistencia con pequeños negocios informales, sino 
también ejercer una presión del mercado sobre esos predios externos, generando incrementos especulativos en los precios del suelo.

\section{IV.4. Terrenos disponibles}

Los terrenos sobre los que se emplaza el barrio pertenecían en su mayoría al Estado Nacional, hasta el cambio de gobierno en 2015, ya sea porque formaban parte del puerto, del ferrocarril o porque eran propiedad de la empresa nacional de combustibles Yacimientos Petrolíferos Fiscales (YPF). Por este motivo, el encargado de administrarlos era la Agencia de Administración de Bienes del Estado (AABE). A partir de ese año, cuando el Gobierno Nacional y de la ciudad pasan a ser del mismo signo político, la AABE establece una articulación directa con el gobierno local y comienza a cederle terreno de propiedad nacional a este último. Entre éstos se encuentran los que ocupa la Villa 31-31 bis y que han sido cedidos con el fin de permitir la regularización. ${ }^{15}$ Otra cantidad importante de tierras será subastada con el objetivo de financiar infraestructura urbana. ${ }^{16}$

Entre los terrenos subastados o en proceso de subasta hay parcelas ubicadas dentro del polígono delimitado para la reurbanización de la Villa, o linderos a éste, que habían sido rezonificadas por la Ley de Urbanización bajo el código "urbanización futura" (UF). Muchos especialistas y habitantes del barrio advirtieron, durante la elaboración del proyecto, sobre el riesgo de no asignar un uso a terrenos vacantes dentro del polígono a reurbanizar, ya que en un futuro podrían ser utilizados para aprovechamiento del mercado. Reclamaban, en cambio, que fuesen utilizados para construir equipamiento de uso común en la nueva urbanización. Al respecto, antes de la sanción de la Ley, el citado coordinador de la SISU reconocía:

${ }^{15}$ https://www.clarin.com/ciudades/nacion-cede-tierras-ciudad-regularizarviviendas-villas-financiar-obras_0_DwpWj47pu.html

${ }^{16} \mathrm{https}$ //www.lanacion.com.ar/buenos-aires/en-dos-anos-ciudad-vendiosuperficie-equivalente-nid2242367?fbclid=IwAR30HkZxguH4AcQok7D6IFoce KT7XJMaGQm5zh44VzKsJ8EeDR5chjUbJPA 
La política de la AABE se parece muchísimo más a la de un agente inmobiliario que a la de un organismo del Estado para la regularización de suelos. Justamente esa política -para mí- entra en contradicción con procesos de reurbanización que se han decidido encauzar a nivel político. Entonces nosotros estamos trabajando en esa tensión: intentando que ese suelo que es dominio de nación sea funcional en su desarrollo al proceso de urbanización que desde la ciudad se decidió emprender. ${ }^{17}$

En la cita precedente, el funcionario asume los riesgos de no proteger los terrenos públicos, dada la posibilidad de que sean privatizados y que esto profundice su mercantilización, ejerciendo presión sobre el barrio.

\section{Conclusiones}

La Villa 31-31 bis o barrio Padre Carlos Mugica tiene una historia de avances y retrocesos entre la erradicación y la permanencia de sus habitantes. Recientemente, las necesidades de renovación y refuncionalización de una zona céntrica y con alto valor potencial, en conjunto con condiciones de coyuntura política local y tendencias urbanísticas a nivel regional, dieron lugar a una acción gubernamental de reurbanización fijada por ley. La norma se basa en la regularización dominial, con instalación de servicios públicos y construcción de viviendas nuevas, pero con pocas mejoras en el equipamiento urbano general y sin el acompañamiento de políticas socioeconómicas complementarias. Algunas de las regulaciones que establece son exclusivas para los habitantes de la Villa 31 y, sumadas a la falta de información sobre componentes centrales del proyecto, podrían exponerlos a una situación de vulnerabilidad frente a otros agentes del mercado.

Dado que estamos ante un fenómeno en desarrollo, los efectos que las acciones públicas analizadas puedan tener en la integración de la Villa a la dinámica urbana sólo pueden ser objeto de hipótesis. En este sentido, Smolka (2002) afirma que en América Latina aún es incipiente el estudio sobre lo que ocurre después de la regularización

${ }^{17} \mathrm{https}$ //www.youtube.com/watch?v=PPdcDHTG-MY\&list=PLRUO32M1 CvrQYpSo2e_xfZHGU2jZAotrV\&index $=4$ 
de asentamientos. Sin embargo, el autor sostiene que, en términos analíticos, pueden ocurrir dos cosas: que los programas sean exitosos -logrando que el área beneficiada se integre al tejido urbano-, lo cual tendrá como resultado que algunos ocupantes permanezcan y otros sean expulsados por la vía del mercado; o que sean mal diseñados y aplicados, lo cual hará que la zona se consolide como irregular y de bajos ingresos ${ }^{18}$ (Smolka, 2002).

Diversos autores sostienen que el desafío, aún no resuelto en este tipo de políticas, es el de generar soluciones que apunten a atender las causas de la informalidad. Sostienen que la sola regularización reviste un carácter curativo si no apunta a revertir el patrón excluyente del régimen urbano, según el cual "mercados de tierras, sistemas políticos y sistemas jurídicos no ofrecen condiciones propias y razonables de acceso al suelo y vivienda para la población de menores recursos" (Almansi, 2009, p. 62). Destaca, en cambio, la necesidad de acompañar las regularizaciones con políticas socioeconómicas más amplias y regulaciones urbanísticas que atiendan las causas que dieron origen a la informalidad (Smolka, 2002; Ostuni y van Gelder, 2009; Fernandes, 2008). De no hacerlo, en el largo plazo los sectores que se desarrollan en la informalidad pueden quedar desposeídos del suelo por acción del mercado, sin ver mejoradas sus condiciones de bienestar ni haberse integrado al modo de producción capitalista (Salazar, 2014).

Cuando el programa aquí presentado culmine, los habitantes del barrio accederán a un título de propiedad -privada e individual-contra el pago de una hipoteca. Destacamos en este punto que la urbanización informal es también informalidad económica, y se explica en parte por la precarización del mercado de trabajo, que dificulta las posibilidades de ahorro familiar (Abramo, 2012). Cargar a los pobres con obligaciones financieras que no puedan afrontar podría tener consecuencias negativas que refuercen los mecanismos que dieron lugar a las villas (Fernandes, 2003). Las disparidades en el acceso a la información entre los habitantes y los agentes del mercado inmobiliario pueden re-

${ }^{18}$ Esto fue lo que ocurrió, por ejemplo, durante los años noventa con el barrio Ramón Carrillo de la Ciudad de Buenos Aires, que fue construido para relocalizar a los ocupantes del ex Albergue Warnes, pero, dada la baja calidad y el poco mantenimiento de la urbanización, hoy toma la forma de un asentamiento informal. 
forzar esta tendencia. Por eso es necesario que la regularización esté acompañada de un proceso integral y participativo en el que se evalúen las necesidades y las capacidades de pago de sus habitantes.

En este sentido, se hace pertinente observar no sólo los cambios producidos dentro del barrio, sino también aquellos que se están generando en el entorno como consecuencia de la inversión en su refuncionalización y renovación, en gran medida impulsadas por el Estado. Sin la intervención del poder público en obras de infraestructura, en los cambios de uso del suelo o en la misma reurbanización de la Villa, los precios de los terrenos que hoy ocupan los emprendimientos inmobiliarios de la zona serían menores. La revalorización de la zona se da en gran medida por la acción del Estado, por lo que éste debería poder recuperar una parte de la ganancia extra generada por su acción (Smolka 2013; Schechinger, 2007). En el caso de estudio, si propietarios y desarrolladores contribuyeran con una porción de las plusvalías generadas, se podría reducir el endeudamiento adquirido, cargar menos las finanzas de los pobres, y generar recursos para acompañar las políticas de reurbanización con otras más amplias. Pero para que ello sea posible, es necesario repensar la idea de la propiedad, no como un derecho sin condicionamientos, sino como un conjunto de derechos (de uso, de construcción, de transformación) que implican obligaciones. De este modo, el Estado podría controlar el ejercicio de cada derecho, recuperar recursos, y así repartir mejor las cargas y beneficios de la ciudad.

Existe también un debate acerca de si la regularización debería implicar la entrega de títulos de propiedad privada plena o si ésta expone a los pobladores a un riesgo, ya que equipara su dominio al de actores con recursos económicos y sociales mucho mayores. Frente a esto, algunos autores proponen alternativas como formas de propiedad colectiva en las que los grupos sean propietarios del suelo, y los individuos o las familias compren o arrienden los inmuebles, según su capacidad de pago. Superar las limitaciones que generan los sistemas de propiedad instituidos, y crear nuevos que contemplen las particularidades existentes en los asentamientos, sigue siendo un desafío.

Para concluir, dado el crecimiento de asentamientos informales en América Latina, consideramos que la seguridad en la tenencia de la tierra se torna una necesidad humanitaria, no sólo por mejorar las 
condiciones de vida de quienes allí residen, sino porque también pagan altos costos por la urbanización informal. En ese camino, la regularización es necesaria pero no debería ser un fin en sí mismo, sino la culminación de un proceso de mejoramiento de las condiciones sociales y habitacionales de la población. Una reurbanización integral implica vincular la legalización de la propiedad y la instalación de servicios, con la provisión de equipamientos de salud, educación, seguridad, etc., y políticas complementarias que mejoren las condiciones socioeconómicas de sus habitantes (Fernandes, 2011). Pero, sobre todo, para generar soluciones no meramente curativas en el acceso al suelo y la vivienda de los sectores populares, se requieren políticas que intervengan en el mercado de tierras y redistribuyan de maneras más equitativas los recursos producidos por la ciudad en su conjunto.

\section{Bibliografía}

Abramo, P. (2008). La producción de las ciudades latinoamericanas: mercado inmobiliario y estructura urbana. Quito, Ecuador: Olacchi.

Abramo, P. (2012). La ciudad informal COM-FUSA: el mercado y la producción de la territorialidad urbana popular. En Clara E. Salazar (coord.), Irregular, suelo y mercado en América Latina (pp. 85-126). Ciudad de México: El Colegio de México, A.C.

Acosta, C. (2019). Regulaciones de suelo, infraestructuras y soportes urbanos, y vivienda social en debates de derecho comparado. En G. Henao González, C. Acosta y J. M. Hofman Quintero (eds.), Manual de Derecho Urbano. Bogotá, Colombia: Universidad del Rosario.

Almansi, F. (2009). La regularización de la tierra en los programas de mejoramiento integral del hábitat. Medio Ambiente y Urbanización, 70(1), 61-88. https://www.ingentaconnect.com/content one/iieal/meda/2009/00000070/00000001/art00006

Azuela, A. (coord.). (2016). La ciudad y sus reglas. Sobre la huella del derecho en el orden urbano. Ciudad de México: Universidad Nacional Autónoma de México / Procuraduría Ambiental y del Ordenamiento Territorial de la Ciudad de México. 
Cravino, M. C. (2009). Entre el arraigo y el desalojo. La Villa 31 de Retiro. Derecho a la ciudad, capital inmobiliario y gestión urbana. Buenos Aires: Universidad Nacional de General Sarmiento.

Cuenya, B. (2011). Grandes proyectos y sus impactos en la centralidad urbana. Cadernos Metrópole, 13(25), 185-212. https://www. redalyc.org/pdf/4028/402837820008.pdf

Cuenya, B. (2012). Grandes proyectos urbanos, cambios en la centralidad urbana y conflictos de intereses. Notas sobre la experiencia argentina. En B. Cuenya, P. Novais y C. Vainer (comps.), Grandes proyectos urbanos. Miradas críticas sobre la experiencia argentina y brasileña (pp. 27-66). Buenos Aires, Argentina: Café de las Ciudades.

Fernandes, E. (2003). Programas de regularización de la tenencia de la tierra urbana y pobreza urbana en Latinoamérica. Revista Vivienda Popular, 12, 5-16.

Fernandes, E. (2008). Consideraciones generales sobre las políticas públicas de regularización de asentamientos informales en América Latina. Revista Latinoamericana de Estudios Urbano Regionales, Eure, 34(102), 25-38. https://scielo.conicyt.cl/pdf/ eure/v34n102/art02.pdf

Fernandes, E. (2011). Regularización de asentamientos informales en América Latina. Cambridge, Estados Unidos: Lincoln Institute of Land Policy.

Fernández Wagner, R. (2008). Los asentamientos informales como cuestión. Revisión de algunos debates. En M. C. Cravino (coord.), Los mil barrios (in)formales. Aportes para la construcción de un observatorio del hábitat popular del Área Metropolitana de Buenos Aires (pp. 13-44). Buenos Aires, Argentina: Universidad Nacional de General Sarmiento

Ibarra, L. (2013). Una política pública entrampada: la regularización de los asentamientos humanos irregulares en suelo de origen social en México. (Tesis de maestría en Políticas Públicas Comparadas, Flacso-México).

Maldonado, M. (2013). Fundamentos del derecho urbanístico en Argentina, Brasil y Colombia. En D. Alfonso Erba (coord.), Definición de políticas de suelo urbano en América Latina. Teoría y práctica (pp. 95-104). Cambridge: Lincoln Institute of Land Policy. 
Ons, M. (2018), La Ley de Urbanización de la Villa 31-31 bis en la Ciudad de Buenos Aires. El debate parlamentario y público en torno a su sanción y aplicación (2007-2015). Quid 16, Revista del Área de Estudios Urbanos, 9, 184-196. https://dialnet.unirioja. es/servlet/articulo? codigo $=6566127$

Ostuni, F. y van Gelder, J. (2009). No sé si legal... ¡pero legítimo es! Percepciones sobre seguridad en la tenencia y títulos de propiedad en barrios informales del Gran Buenos Aires. En M. C. Cravino (coord.), Los mil barrios (in)formales. Aportes para la construcción de un observatorio del hábitat popular del Área Metropolitana de Buenos Aires (pp. 201-230). Buenos Aires, Argentina: Universidad Nacional de General Sarmiento.

Salazar, C. (2012). Los ejidatarios en el control de la regularización. En Clara E. Salazar (coord.), Irregular, suelo y mercado en América Latina (pp. 265-306). Ciudad de México: El Colegio de México, A.C.

Salazar, C. (2014). El puño invisible de la privatización. Territorios, 30, 69-90. https://revistas.urosario.edu.co/index.php/territorios/ article/view/3111

Salazar, C. (2020). Mercado de tierra ejidal en la periferia urbana: transformación institucional en un contexto de inequidad social. En G. Torres-Mazuera y K. Appendini (coords.), La regulación imposible (i)legalidad e (i)legitimidad en los mercados de tierra en México al inicio del siglo XXI (pp. 241-282). Ciudad de México: El Colegio de México, A.C.

Sassen, S. (1998). Ciudades en la economía global: enfoques teóricos y metodológicos. Revista Latinoamericana de Estudios Urbano Regionales, Eure, 24(71), 5-25. https://www.eure.cl/index. $\mathrm{php} /$ eure/article/view/1158

Schechinger, C. (2007). Algunas reflexiones sobre el financiamiento de las ciudades con suelo urbano. Cuaderno de Docencia. Lincoln Institute of Land and Policy.

Smith, N. (2007). La nueva frontera urbana. Ciudad revanchista y gentrificación. Madrid, España: Traficantes de sueños.

Smolka, M. (2002). Regularización de la ocupación del suelo urbano. El problema que es parte de la solución, la solución que es 
parte del problema. Cuaderno de Docencia. Lincoln Institute of Land and Police.

Smolka, M. (2013). Implementación de la recuperación de plusvalias en América Latina. Políticas e instrumentos para el desarrollo urbano. Cambridge: Lincoln Institute. https://www. lincolninst.edu/sites/default/files/pubfiles/implementacionrecuperacion-de-plusvalias-full_0.pdf

Smolka, M. y Furtado, F. (2001). Recuperación de plusvalías en Latinoamérica: ¿bravura o bravata? Cambridge: Lincoln Institute of Land Policy.

Wacquant, L. (2007). Los condenados de la ciudad. Gueto, periferias y Estado. Buenos Aires, Argentina: Siglo XXI.

\section{Acerca de la autora}

Melina Ons es licenciada y profesora en Sociología, especialista en Planificación y Gestión de Políticas Sociales, y cursa el Doctorado en Ciencias Sociales, todo por la Universidad de Buenos Aires. Realizó una estancia de investigación en el Centro de Estudios Demográficos, Urbanos y Ambientales de El Colegio de México en el año 2019. Actualmente desarrolla sus actividades de investigación en el Instituto del Conurbano de la Universidad Nacional de General Sarmiento, en el marco del sistema del Consejo Nacional de Investigaciones Científicas y Técnicas de Argentina. Su línea de investigación se enfoca en las acciones del Estado en áreas donde coexisten grandes desarrollos inmobiliarios privados y de infraestructura pública con asentamientos informales, durante los años 2007-2022 en la Ciudad y la Provincia de Buenos Aires; específicamente en lo referente al manejo del suelo y sus posibles consecuencias sobre la dinámica del mercado y la población residente. ORCID: https://orcid. org/0000-0001-8951-3897

Recepción: 9 de julio de 2019. Aceptación: 23 de octubre de 2019. 